\title{
Curbside Consultation in Neuro-Ophthalmology-49 Clinical Questions. Eds: Andrew G. Lee, Paul W. Brazis and Lanning B. Kline. ISBN: 978-1-55642-840-1 SLACK Incorporated
}

\author{
Valéria Nagy
}

Received: 2 December 2009 / Accepted: 10 December 2009 /Published online: 19 January 2010

(C) Springer-Verlag 2010

The title of this book is of interest to the readers (ophthalmologists). Although the book is relatively short, it covers many topics of neuro-ophthalmology. The softbound book is divided into 49 chapters/questions, consisting of references presenting the latest scientific knowledge in neuro-ophthalmology. The question and answer type format is unique, interesting and user-friendly. The chapters are well-structured, beginning with a case report (one of the personal cases of the author of the given chapter), followed by the analysis of the problem, and finally a conclusion or summary and bibliography. Tables and black and white or color figures make the book more interesting to the readers.

The three editors and 61 co-authors are focused on mainly clinical problems/questions of neuro-ophthalmology. The co-authors are outstanding clinicians in the given field, working in famous hospitals in the USA. The themes of the text are timely, important and thought-provoking.

The book is recommended by the authors to clinicians, to specialists and maybe to residents. Comparable text has not been published on this theme lately. The authors' aim is that the book should be suitable for four requirements, i.e., current, concise, credible (evidence-based) and clinically relevant (practical).

Their analysis is important, and shows real problems which could meet our daily routine/practice. The book introduces cases of optic nerve tumors, non-arteritic anterior ischemic and giant cell arteritis, optic neuropathy, optic neuritis, traumatic optic neuropathy, papilledema,

\section{Nagy $(\bowtie)$}

Department of Ophthalmology,

Medical and Health Science Center, University of Debrecen, Debrecen, Hungary

e-mail: vnagy@med.unideb.hu ocular myasthenia gravis, thyroid eye disease, nystagmus, anisocoria and pupillary reflex defects, toxic neuropathy, optic atrophy, nerve palsies, and blepharospasm. The authors review the most important and remarkable rare diseases such as carotid cavernous fistula and non-organic visual loss. It presents interesting cases of migraine patients and visual loss after spinal surgery.

The authors analyze in a "curbside consultation"clinical questions form their subjects of clinical practice. They demonstrate the modern well-defined neuroimaging screening examinations such as computed tomography (CT), magnetic resonance imaging (MRI) with/or without gadolinium, and CT angiogram. The book analyses the benefit of the neuromaging examinations and provides readers with guidance for resolving problems in their clinical practice. The authors conclude with practical advice to physicians for the future based on their experiences. The authors' questions/answers demonstrate in their opinion the exact management and treatment. They present the adequate therapy for neuro-ophthalmological diseases, and emphasize those cases in which surgical treatment is recommended. Authors show those cases for which there is no treatment at the moment. Clinicians highlight the most important circumstances which could cause exacerbation of the disease.

The book is practical for specialists, ophthalmologists and residents in their clinical practice, and helps to solve problems in neuro-ophthalmology. Its content, form, and style are clear and obvious. The book gives ideas for thought and practical advice to specialists and beginners (residents) as well. The book can be recommended for residents of ophthalmology too. It is evident that the aims of the editors and authors are certainly met. 